\title{
Cálculos teóricos para elucidação eletrônica de barreiras rotacionais: teoria e aplicações
}

\author{
Murielly Fernanda Ribeiro Bihain ${ }^{\mathrm{a}}{ }^{\circ}$, Raissa Santos Sousa ${ }^{\mathfrak{\oplus}}$, Sílvio Quintino de Aguiar Filho ${ }^{\mathrm{a}}$, \\ Douglas Henrique Pereira ${ }^{a^{*}(D)}$
}

\footnotetext{
${ }^{\mathrm{a}}$ Universidade Federal do Tocantins, Brasil

*Autor correspondente (doug@uft.edu.br)
}

\section{N F O}

\section{Keywords}

theoretical calculations stereoeletronic effects EDA

QTAIM

NBO

\begin{abstract}
A B S T R A C T
Theoretical calculations for electronic elucidation of rotational barriers: theory and applications.

Theoretical calculations of electronic structure are tools of theoretical chemistry. Its importance extends from rotation analyses, hyperconjugation, steric effects on orbital location calculations, energy level and interaction between ligand and orbitals antiligantian. Theories like Natural Bond Orbital (NBO), Quantum Theory of Atoms in Molecules (QTAIM), e Energetic Decomposition Analysis (EDA), are examples of theoretical tools that describe the electronic structure of molecules and solids. In this context, the aim of the article was to describe the theoretical approaches of NBO, EDA and QTAIM, briefly with its applications in the chemical system hydrazine and in theoretical works found in the literature that reinforce the elucidation off stereoelectronic effects.
\end{abstract}

\section{R E S U M O}

Cálculos teóricos de estrutura eletrônica são ferramentas da química teórica. Sua importância se estende desde a análises de rotação, hiperconjugação, efeitos estéricos a cálculos de localização de orbitais, nível de energia e interação entre orbitais ligantes e antiligantes. As teorias como Natural Bond Orbital (NBO), Quantum Theory of Atoms in Molecules (QTAIM), e Energetic Decomposition Analysis (EDA), são exemplos de ferramentas teóricas que descrevem a estrutura eletrônica de moléculas e sólidos. Neste contexto o objetivo do artigo foi descrever as abordagens teóricas do NBO, EDA e QTAIM de forma breve com suas aplicações no sistema químico hidrazina e em trabalhos teóricos encontrados na literatura que reforçam a elucidação dos efeitos estereoeletrônicos. 


\section{INTRODUÇÃO}

A análise de ligações sigmas $(\sigma)$ e suas rotações levaram a ciência a um vasto campo de descobertas que permite analisar o comportamento de um sistema molecular (de Aguiar Filho et al., 2019). O estudo das rotações moleculares é fundamental para entender mudanças de conformações moleculares, que estão estreitamente relacionadas com problemas de grande importância nas áreas da química, biologia e bioquímica (Liu, 2013; Pereira et al., 2014; de Aguiar Filho et al., 2019).

A preferência conformacional de sistemas químicos pode ser justificada pelas barreiras rotacionais, nas quais os efeitos esteroeletrônicos regem suas conformações. Os principais efeitos responsáveis pelas alturas das barreiras rotacionais são os efeitos estéricos e os provenientes da movimentação da densidade eletrônica pela estrutura do sistema, que podem ter diferentes naturezas, tais como hiperconjugação e efeito anomérico. (Mulliken, e Brown, 1941; Juaristi e Cuevas, 1992; Liu, 2007; de Aguiar Filho et al., 2019).

Neste contexto, é importante destacar que o estudo de barreiras rotacionais permite elucidar, a partir de uma perspectiva energética e estrutural, a preferência físico-química por um dado confôrmero, principalmente para moléculas orgânicas (Liu, 2013; de Aguiar Filho et al., 2019). Este conceito tem sido aplicado, por exemplo, em estudos sobre a reatividade química, dobramento e desdobramento de proteínas envolvidas na doença da vaca louca e em análises conformacionais de polímeros (de Aguiar Filho et al., 2019; Reis et al., 2019).

Alinhado aos resultados experimentais, há cálculos computacionais que empregam diferentes teorias, como os métodos ab initios (Hehre, 1976), semi-empíricos (Cukrowski, 2015), os baseados na teoria do funcional de densidade (do inglês, Density Functional Theory, DFT) (Morgon e Custodio, 1995) e os métodos compostos que são amplamente empregados com o intuito de entender e explicar a altura de barreiras rotacionais dos mais diferentes sistemas químicos (de Aguiar Filho et al., 2019; Pereira et al., 2014). Entretanto, a elucidação estrutural dos efeitos estereoeletrônicos, que por sua vez regem o valor de uma barreira rotacional, pode ser feita a partir da função de onda dos métodos descritos acima utilizando diferentes aproximações para definir e compreender as especificidades dos sistemas químicos (Liu, 2013; Cukrowski, 2015). Algumas abordagens amplamente empregadas são: a) Orbitais Naturais de Ligação (do inglês, Natural
Bond Orbitals, NBO) (Foster e Weinhold, 1980; Glendening et al., 2012), b) Análise de Decomposição Energética (do inglês, Energy Decomposition Analysis, EDA) (Morokuma, 1971) e c) Teoria Quântica de Átomos em Moléculas (do inglês, Quantum Theory of Atoms in Molecules, QTAIM) (Bader e Nguyen-Dang, 1981).

Frente a importância das barreiras rotacionais, o trabalho tem como objetivo descrever, de modo simplificado, as abordagens teóricas dos métodos NBO, EDA e QTAIM com ênfase em sua formulação inicial, realizando uma aplicação em um sistema químico conhecido, a hidrazina $\left(\mathrm{H}_{2} \mathrm{NNH}_{2}\right)$, e abordar alguns trabalhos da literatura sobre a elucidação dos efeitos esteroeletrônicos das metodologias descritas.

\section{MATERIAL E MÉTODOS}

A fim de verificar de forma prática a aplicação das metodologias, um estudo foi realizado com a molécula de hidrazina $\left(\mathrm{H}_{2} \mathrm{NNH}_{2}\right)$. Para o estudo, empregou-se a teoria do funcional de densidade com funcional híbrido B3LYP (Parr e Yang, 1989; Dreizler, 1990) e a função de base $6-31+G(d, p)$, tanto para a otimização de geometria quanto para os cálculos NBO, QTAIM e EDA. Nos cálculos de otimização variou-se o ângulo diedro de $0^{\circ}$ até $360^{\circ}$ em intervalos de $10^{\circ}$. Os cálculos das alturas das barreiras rotacionais foram determinados a partir das geometrias livres. Algumas estruturas foram desenhadas no programa GaussView 5.09 (Dennington et al., 2009) e os cálculos de otimização, NBO e EDA realizados no programa Gaussian 09 (Frisch, et al., 2009). A análise QTAIM foi realizada a partir do programa AIMAll (Keith, 2017). Para a EDA, os componentes de energia para a análise foram obtidos do programa Gaussian 09 com o $\operatorname{iop}(5 / 33=1)$. Para a análise foi considerada a diferença de energia entre a estrutura mais estável em $30^{\circ}$ subtraído dos máximos, $110^{\circ}$ e $290^{\circ}$.

\section{Abordagens Teóricas (Orbital Natural de Ligação)}

Os Orbitais Naturais de Ligações, são oriundos de análises dos orbitais naturais realizado por Frank Weinhold ao qual se inspirou no trabalho de PerOlov Löwdin (Löwdin, 1955). Suas pesquisas são resultantes de uma solução de não adequação simplificada da visão de Lewis em relação a ligação covalente dentro da Teoria do Orbital Molecular (Löwdin, 1955; Foster e Weinhold, 1980). 
Os estudos sobre NBO partem da compreensão de uma sequência de bases de orbitais naturais atômicos (do inglês, Natural Atomic Orbitals, NAOs), orbitais naturais híbridos (do inglês, Natural Hybrid Orbitals, NHOs), orbitais naturais de ligação (NBOs) e orbitais moleculares naturais localizados (do inglês, Natural Localized Molecular Orbitals, NLMOs) seguindo uma ordem de hierarquia. Tem como objetivo avaliar os efeitos de deslocalização eletrônica de átomos ou grupos atômicos permitindo prever hibridização, efeitos estéricos, transferência de cargas, dentre outros (Glendening et al., 2012).

As análises de NBO originaram-se através de técnicas empregadas em estudos relacionados à hibridização e efeitos de covalência em funções de onda poliatômicas e expressam propriedades moleculares com caráter de uma estrutura natural de Lewis (Weinhold, 2012).

\section{- Teoria}

O método NBO envolve os termos de pares de elétrons ligantes, ligações químicas localizadas, deslocalizadas e ainda pares de elétrons isolados como unidade básica molecular. Os orbitais naturais de ligação são calculados com a máxima ocupação eletrônica, em que há a consistência de uma conversão de uma função de onda inicial para outras funções de onda das sequências de conjuntos: orbitais naturais atômicos (NAOs), orbitais naturais híbridos (NHOs), orbitais naturais de ligação (NBOs) e orbitais moleculares naturais localizados (NLMOs) que está interposto aos orbitais atômicos (do inglês, Atomic Orbital, AO) e aos orbitais moleculares (do inglês, Molecular Orbital, MO) (Glendening et al., 2012).

Quando há conjuntos localizados, os mesmos podem ser modificados para orbitais naturais localizados (do inglês, Natural Localized Orbitals, NOs) ou retomar para orbitais moleculares canônicos (MOs). Essas modificações podem gerar orbitais que são ocupados em múltiplas direções, orbitais antiligantes e de Rydberg, que podem atuar como armazenadores de transferências de cargas eletrônicas que varia com a conformação molecular. Dessa forma, as cargas atômicas são exemplificações de suma importância nos estudos conformacionais podendo ser escritos por meio da densidade eletrônica que abrange os átomos que participam da interação (Vorobyov et al., 2002; Glendening et al., 2012).

Existem os NBOs do tipo que não aderem à estrutura natural de Lewis que descrevem os efeitos de deslocalização. As deslocalizações eletrônicas são quantitativamente analisadas pela remoção de orbitais antiligantes e de Rydberg, que resulta em estruturas de Lewis idealizadas que podem obter a contribuição dos efeitos estérico, hiperconjugativos e ainda a estimativa de força de interação e deslocamento de carga por análise da energia de perturbação de segunda ordem (Vorobyov et al., 2002; Raissi et al., 2009).

Os cálculos de NBO são feitos por etapas e são implementados em uma variedade de pacotes de cálculos de estrutura eletrônicas como Gaussian, GAMES, MOPAC dentre outros (Glendening et al., 2012). Podem ser realizados utilizando diferentes níveis de teorias como os métodos ab initio, baseados em DFT e semi-empíricos.

Neste contexto, as análises de NBO procuram todas as possíveis formas de representar as ligações e pares de elétrons isolados para um padrão de ligação idealmente variável que procura a máxima ocupação.

\section{Abordagens Teóricas (Teoria Quântica de Átomos em Moléculas)}

A Teoria Quântica de Átomos em Moléculas (do inglês, Quantum Theory of Atoms in Molecules, QTAIM) de Richard F. W. Bader e colaboradores surgiu em 1990 com o propósito de descrever a natureza das ligações químicas de sólidos, moléculas e também interações intramoleculares e intermoleculares (Oliveira et al., 2010). Pela QTAIM, as ligações podem ser interpretadas como um compartilhamento de elétrons (covalente), intermediária (parcialmente covalente) e interações de camada fechada, como van der Waals, iônica, metálica, entre outras (Vener et al., 2007). Abaixo, segue uma breve descrição da teoria.

\section{- Teoria}

Na QTAIM, tem-se que os observáveis quânticos de um dado sistema químico estão contidos na densidade eletrônica, definida como $\rho(\mathrm{r})$ (Oliveira et al., 2010). A densidade eletrônica por sua vez pode ser representada como:

$$
\rho(\mathrm{r})=N \int \mathrm{d} \tau \psi^{*} \psi
$$

em que $\mathrm{N}$ é o número de elétrons, $\mathrm{d} \tau$ é o volume do sistema químico considerado, $r$ são as coordenadas espaciais do elétron e $\psi$ é a função de onda que, na mecânica quântica (Kumar et al., 2016), contém 
todas as informações relevantes para um dado sistema (Oliveira et al., 2010).

A condição básica para se determinar a topologia molecular do sistema químico é dividir, ou particionar o sistema em fragmentos ou subsistemas químicos (Oliveira et al., 2010). Neste contexto o átomo pode ser então definido em termos desses fragmentos atômicos, sendo então limitado pelos mesmos, que por sua vez, definem o que é chamado de bacia atômica (Bader e Nguyen-Dang, 1981). Entender a forma do particionamento dos átomos é importante, pois, este é o conceito fundamental para se entender a teoria, uma vez que as demais considerações surgem desse princípio (Popelier e Bader, 1992).

Os fragmentos são delimitados entre os átomos que compõe o sistema químico e são exatamente nestes pontos que estão localizados os chamados subsistemas químicos (Bader, 1985). Esses subsistemas químicos se tratam de superfícies, denominadas superfícies interatômicas.

As superfícies devem satisfaz a equação 2:

$$
\nabla \rho(\mathrm{r}) \cdot \mathrm{n}(\mathrm{r})=0
$$

em que n(r) é um vetor unitário normal a superfície em r (Bader e Nguyen-Dang, 1981). As superfícies são bem definidas pelo o que é chamado de atrator nuclear, que é propriamente o núcleo do sistema químico (Oliveira et al., 2010).

$\mathrm{Na}$ mesma região molecular dos núcleos, existem máximos locais de densidade eletrônica, que são justamente os atratores nucleares (Anderson et al., 2019). Além disso, a densidade eletrônica pode exibir também regiões de mínimo local ou ponto de sela, no qual esses pontos especiais são chamados de pontos críticos (no inglês, critical point, $\mathrm{CP}$ ) que aparecem na região entre dois atratores (Kumar et al., 2016). Em um CP, tem-se que as primeiras derivadas da densidade eletrônica desaparecem, ou seja, $\nabla \rho=0$, e $\nabla \rho$ é dado pela expressão 3 (Kumar et al., 2016).

$$
\nabla \rho(\mathrm{r})=\mathrm{i} \frac{\partial \rho}{\partial x}+\mathrm{j} \frac{\partial \rho}{\partial y}+\mathrm{k} \frac{\partial \rho}{\partial z}
$$

Dado que um CP é formado na região entre dois núcleos, admite-se que os mesmos estão ligados entre si, formando assim uma ligação química como descrito na Figura 1 (Oliveira et al., 2010).

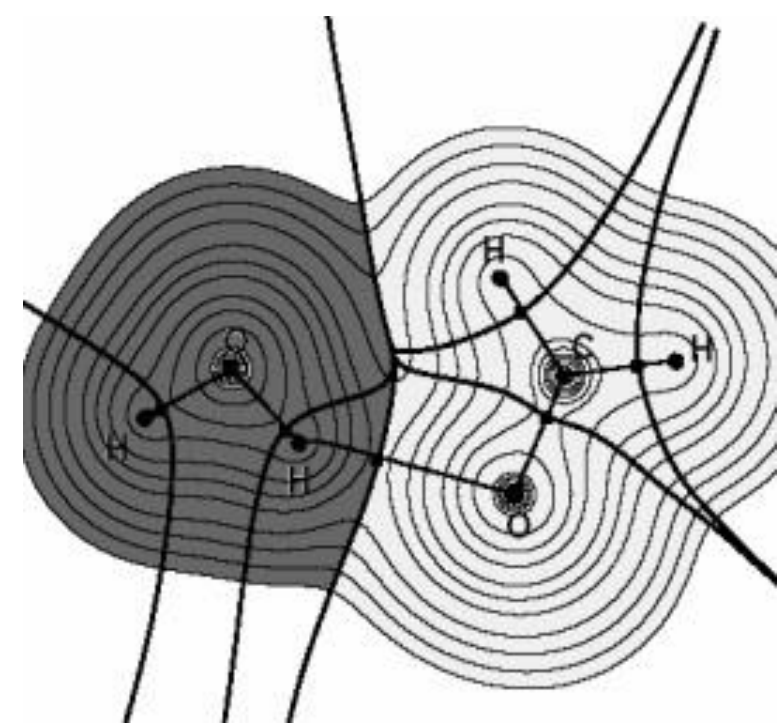

Figura 01 - Em preto a molécula de água, em branco a molécula de metanal. As linhas grossas escuras representam as superfícies interatômicas (Popelier, 2005).

Os CPs podem admitir três naturezas diferentes como representado na Figura 2. A primeira é chamada de ponto crítico de ligação (do inglês, bond critical point, $\mathrm{BCP}$ ) que é simplesmente o ponto que une dois atratores; ponto crítico de anel (do inglês, ring critical point, $\mathrm{RCP}$ ) que se forma no interior de estruturas cíclicas e ponto crítico de gaiola (do inglês, cage critical point, CCP) que se forma no interior de estruturas anelares que se unem de tal maneira que uma gaiola seja formada, se tratando, assim, de um conjunto de estruturas cíclicas (Anderson et al., 2019).

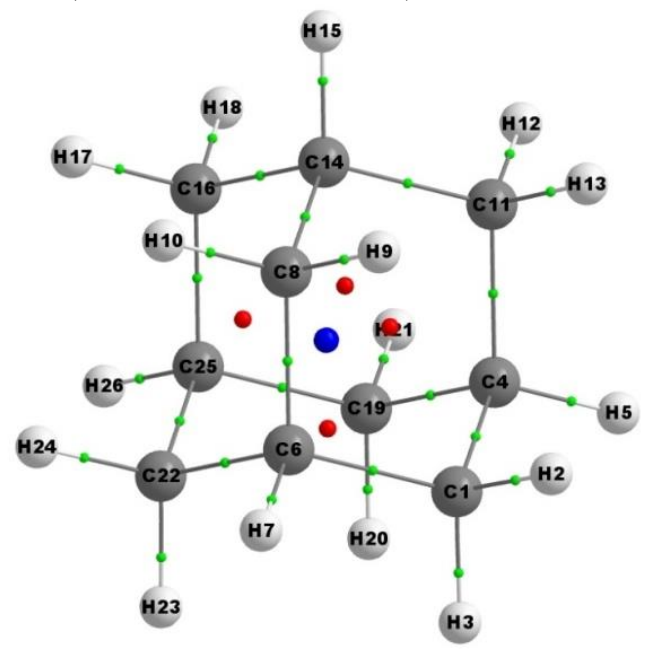

Figura 02 - Estrutura da adamantina gerada a partir do programa AIMAll. Pontos verdes representam os BCPs, esferas vermelhas os RCPs e a esfera em azul o CCP. 
Distinguir entre pontos máximo, mínimo e de sela só é possível se as derivadas de segunda ordem da densidade eletrônica, $\nabla^{2} \rho$, forem consideradas (Anderson et al., 2019). A equação 4 a seguir define matematicamente $\nabla^{2} \rho$.

$$
\nabla^{2} \rho(r)=\frac{\partial^{2} \rho}{\partial x^{\prime}}+\frac{\partial^{2} \rho}{\partial y^{\prime}}+\frac{\partial^{2} \rho}{\partial z^{\prime 2}}=\lambda_{1}+\lambda_{2}+\lambda_{3}
$$

Os valores $\lambda_{1}, \lambda_{2}$ e $\lambda_{3}$ são autovalores dos principais eixos de curvatura da densidade eletrônica com relação aos eixos x, y e z (Kumar et al., 2016). $\nabla^{2} \rho$ é conhecido como laplaciana da densidade eletrônica e, a partir deste parâmetro, algumas considerações podem ser feitas. Para $\nabla^{2} \rho<0$, tem-se que a carga eletrônica é concentrada localmente e em termos de ligação química significa que a natureza dessa concentração de carga reflete numa ligação covalente ou insaturada, se localizando na região em que o CP é formado (Bader, 1985). Para $\nabla^{2} \rho>$ 0 , tem-se que a densidade de carga se encontra dispersa ao longa da superfície interatômica, o que significa que a densidade de carga tende a se concentrar nos átomos ao invés de se concentrar nos CPs, o que caracteriza ligações iônicas e interações de camada fechada (Bader, 1985).

\section{Abordagens Teóricas (Análise de Decomposição Energética)}

A Análise de Decomposição Energética (EDA) foi desenvolvida por Morokuma (Morokuma, 1971) e Ziegler e Rauk (Ziegler e Rauk, 1977). É uma ferramenta importante e poderosa, pois permite conectar conceitos importantes da mecânica quântica com uma interpretação simples do conceito de ligação química (Zhao et al., 2018).

A EDA decompõe a energia de interação instantânea $\left(\Delta \mathrm{E}_{\text {int }}\right)$ entre dois fragmentos A-B de uma molécula A-B em três termos bem definidos que podem ser interpretados quimicamente. Os termos são 1) interação eletrostática clássica entre os fragmentos, $\left.\left(\Delta \mathrm{E}_{\text {elet }}\right), 2\right)$ repulsão de Pauli entre os orbitais ocupados dos fragmentos interagentes ( $\left.\Delta \mathrm{E}_{\text {Pauli }}\right)$ e 3$)$ interações entre orbitais moleculares ocupados de um fragmento $\left(\Delta \mathrm{E}_{\text {orb }}\right)$.

Atualmente existem diversos métodos de decomposição energética, como decomposição energética natural (do inglês, Natural Energy Decomposition Analysis, NEDA), EDA utilizando orbitais moleculares totalmente localizados (Khaliullin et al., 2007) e EDA com partição de Orbitais Naturais para Valência Química (do inglês, Natural Orbital for Chemical Valence, NOCV) (Mitoraj e Michalak, 2007; Mitoraj et al., 2009).
A EDA é utilizada para estudar os mais diferentes sistemas químicos e o conceito abordado aqui será entre a diferença de energia de conformações.

\section{- Teoria}

Para o presente trabalho apenas a EDA usando a DFT foi elucidada. Neste contexto a energia total de um átomo e/ou uma molécula, pode ser descrita como:

$$
E[\rho]=E_{s}[\rho]+E_{e}[\rho]+E_{q}[\rho]
$$

em que, $E_{s}[\rho], E_{e}[\rho]$ e $E_{q}[\rho]$ são as contribuições independente para a energia dos efeitos estérico, eletrostático e quântico, respectivamente. $\mathrm{Na}$ DFT (Parr e Yang, 1989) a energia total pode ser particionada em cinco termos, equação 6:

$$
E[\rho]=T_{s}[\rho]+V_{n e}[\rho]+J[\rho]+V_{n n}[\rho]+
$$

$T_{s}[\rho]$ é o termo cinético não interagente, $V_{n e}[\rho]$ atração núcleo-elétron, $J[\rho]$ repulsão de Coulomb entre elétrons, $V_{n n}[\rho]$ repulsão núcleo-núcleo e $V_{x c}[\rho]$ o termo de troca e correlação, respectivamente. Três termos da equação 6 são de natureza eletrostática, $J[\rho], V_{n e}[\rho] \mathrm{e} V_{n n}[\rho]$ podendo ser reorganizada em um único termo de natureza eletrostática denominado $E_{e}[\rho]$, equação 7 :

$$
E_{e}[\rho]=V_{n e}[\rho]+J[\rho]+V_{n n}[\rho]
$$

Reescrevendo a equação (6) tem-se que:

$$
E[\rho]=T_{s}[\rho]+V_{x c}[\rho]+E_{e}[\rho]
$$

Cada um desses componentes tem um significado físico diferente e essa alternativa de composição de energia é convencional. Outra maneira de particionar a energia foi descrita por Liu (Liu, 2007) e apresenta uma perspectiva físico-química conforme a equação 9 .

$$
\Delta \mathrm{E}=\Delta \mathrm{E}_{\mathrm{s}}+\Delta \mathrm{E}_{\mathrm{e}}+\Delta \mathrm{E}_{\mathrm{q}}
$$

Os termos $E_{\mathrm{s}}, \mathrm{E}_{\mathrm{e}}$ e $\mathrm{E}_{\mathrm{q}}$ representam contribuições energéticas de três efeitos físico-químicos independentes: efeitos quânticos estéricos $\left(\mathrm{E}_{\mathrm{s}}\right)$, eletrostáti$\cos \left(E_{e}\right)$ e efeitos fermiônicos quânticos $\left(E_{q}\right)$, respectivamente. $\mathrm{Na}$ equação 10 , a componente de energia associado ao efeito estérico é simplesmente a energia cinética de Weizsäcker, 


$$
E_{s}=T_{w}[\rho]=\frac{1}{8} \int \frac{\left|\nabla_{\rho}(r)\right|^{2}}{\rho(r)} d r
$$

$\rho(\mathrm{r})$ e $\nabla \rho(\mathrm{r})$ são a densidade eletrônica e seu gradiente respectivamente. Os efeitos fermiônicos quânticos, equação 11, podem ser descritos como:

$$
\mathrm{Eq}=\mathrm{T}_{\mathrm{S}}-\mathrm{T}_{\mathrm{W}}+\mathrm{E}_{\mathrm{xc}}
$$

A nova formulação tem seu próprio significado físico e foi aplicada a vários sistemas moleculares (Liu, 2013; Liu et al., 2008; Liu e Schauer, 2015; Liu et al., 2017).

\section{Estrutura eletrônica da barreira rotacional da hidrazina}

Os cálculos das barreiras rotacionais da hidrazina, as análises das conformações mais estáveis e menos estáveis, bem como as análises NBO, QTAIM e EDA foram realizadas para elucidar a aplicação das metodologias de cálculo. A variação do ângulo diedro se deu na ligação sigma $(\sigma)$ nitrogênio-nitrogênio (N-N) da estrutura variando o diedro de $0^{\circ}$ a $360^{\circ}$ em intervalos de $10^{\circ}$, Figura 03 .

Pela Figura 03 pode-se identificar que a estrutura possui dois máximos de energia em $110^{\circ}$ e $290^{\circ} \mathrm{e}$ um mínimo de energia em $30^{\circ}$. A representação estrutural de cada confôrmero é apresentada na Fi- gura 04. Os valores das barreiras rotacionais encontrados são $10,01 \mathrm{kcal} \mathrm{mol}^{-1} \mathrm{e} 2,95 \mathrm{kcal} \mathrm{mol}^{-1}$ para o método B3LYP/6-31+G(d,p). Os valores experimentais das barreiras rotacionais são $8,57 \mathrm{kcal} \mathrm{mol}^{-}$ ${ }^{1}$ e 3,15 kcal mol${ }^{-1}$ (Pereira et al., 2014, Tsuboi M, 1974) e assim é possível inferir que os cálculos teóricos utilizados no trabalho reproduzem adequadamente as barreiras rotacionais da hidrazina.

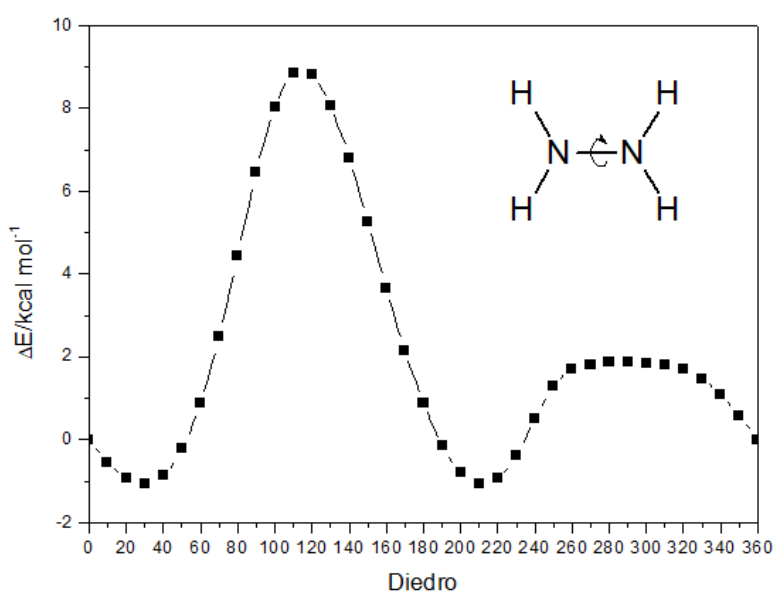

Figura 03 - Gráfico das energias relativas da hidrazina calculadas a partir do ângulo diedro em nível B3LYP/6-31+G(d,p). a)

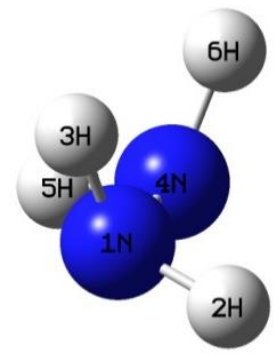

b)

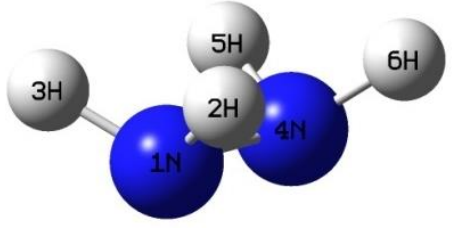

c)

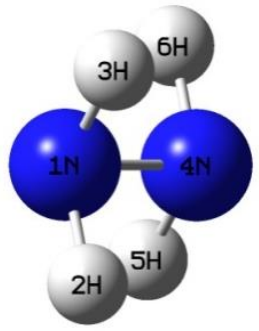

Figura 04 - Representação estrutural da hidrazina, em a) diedro de $30^{\circ}$, b) diedro de $110^{\circ}$ e c) diedro de $290^{\circ}$.

A partir do perfil da barreira rotacional da molécula de hidrazina, é possível elucidar quais são os efeitos estereoeletrônicos que regem as conformações da hidrazina usando as teorias NBO, QTAIM e EDA.

\section{Estrutura eletrônica da barreira rotacional da hidrazina (Análise NBO da hidrazina)}

A análise de NBO permite elucidar quais são as interações que ocorrem entre os orbitais formalmente ocupados e desocupados nos diedros que exibiram mínimos e máximos de energia, respectivamente $30^{\circ}, 110^{\circ}$ e $290^{\circ}$. A Tabela 1 , a seguir, mostra as principais interações entre os orbitais para a hidrazina. Os resultados indicam que há um maior número de interações entre os orbitais ligantes e antiligantes para o diedro de $110^{\circ}$, quando comparado aos diedros de $30^{\circ}$ e $290^{\circ}$, sendo oito interações $\sigma \mathrm{N}-\mathrm{H} \rightarrow \sigma^{*} \mathrm{~N}-\mathrm{H}$ e quatro interações $\mathrm{n} \pi \mathrm{N} \rightarrow \sigma^{*} \mathrm{~N}-\mathrm{H}$. Em $30^{\circ}$ existe duas interações $\sigma \mathrm{N}-\mathrm{H} \rightarrow \sigma^{*} \mathrm{~N}-\mathrm{H}$ e quatro interações dos pares isolados do nitrogênio com os orbitais $\sigma^{*} \mathrm{~N}-\mathrm{H}$ da estrutura. Por fim, em $290^{\circ}$ pode-se observar quatro interações $\sigma \mathrm{N}-\mathrm{H} \rightarrow \sigma^{*} \mathrm{~N}-\mathrm{H}$ e nenhuma interação do tipo $\mathrm{n} \pi \mathrm{N} \rightarrow \sigma^{*} \mathrm{~N}-\mathrm{H}$.

Esses resultados mostram que, para o diedro em 
$30^{\circ}$ a repulsão de Pauli é mais significativa, resultado da movimentação da nuvem eletrônica, tanto que, a geometria só permite que os orbitais ligantes e antiligantes do N1-H2 e N4-H5 interajam, não havendo interação entre os orbitais ligantes e antiligantes nas ligações N1-H3 e N2-H6 (vis Figura 4a). Já no confôrmero em $110^{\circ}$, pela análise da geometria, é possível inferir observações de interações entre os orbitais ligantes e antiligantes de todos os constituintes da hidrazina. Dessa forma, pode-se atribuir que: os efeitos de deslocalização da densidade eletrônica são favorecidos pela geometria e o hidrogênio, que por ser um átomo pequeno, faz com que a repulsão de Pauli, neste caso, seja pouco pronunciada (vis Figura 3b). Por fim, o confôrmero em de $290^{\circ}$ possui apenas metade das interações que a estrutura no diedro em $110^{\circ}$, pois a geometria escalonada permite apenas interações entre os orbitais ligantes e antiligantes das ligações N1-H3 e N4-H5 e entre os orbitais ligantes e antiligantes das ligações simples N1-H2 e N4-H6 (vis Figura 3c).

Tabela 1 - Energia de estabilização de segunda ordem $\left(\Delta \mathrm{E}^{2}\right)$, diferença de energia entre os orbitais $\left(\varepsilon_{\mathrm{i}}-\varepsilon_{\mathrm{j}}\right)$, e elementos da matriz de Fock $(\mathrm{F}(\mathrm{i}, \mathrm{j}))$, para as conformações em $30^{\circ}, 110^{\circ}$ e $290^{\circ}$ da molécula de hidrazina.

\begin{tabular}{|c|c|c|c|c|}
\hline NBO doador (i) & NBO receptor (j) & $\Delta \mathrm{E}^{2} \mathrm{kcal} \mathrm{mol}^{-1}$ & $\varepsilon_{\mathrm{i}}-\varepsilon_{\mathrm{j}}$ & $F(i, j)$ u.a \\
\hline \multicolumn{5}{|c|}{ Ângulo diedro de $30^{\circ}$} \\
\hline$\sigma \mathrm{N} 1-\mathrm{H} 2$ & $\sigma^{*} \mathrm{~N} 4-\mathrm{H} 5$ & 1,88 & 1,10 & 0,041 \\
\hline$\sigma \mathrm{N} 4-\mathrm{H} 5$ & $\sigma^{*} \mathrm{~N} 1-\mathrm{H} 2$ & 1,88 & 1,10 & 0,041 \\
\hline $\mathrm{n} \pi \mathrm{N} 1$ & $\sigma^{*} \mathrm{~N} 4-\mathrm{H} 5$ & 1,57 & 0,80 & 0,032 \\
\hline $\mathrm{n} \pi \mathrm{N} 1$ & $\sigma^{*} \mathrm{~N} 4-\mathrm{H} 6$ & 5,38 & 0,78 & 0,058 \\
\hline $\mathrm{n} \pi \mathrm{N} 4$ & $\sigma^{*} \mathrm{~N} 1-\mathrm{H} 2$ & 1,57 & 0,80 & 0,032 \\
\hline $\mathrm{n} \pi \mathrm{N} 4$ & $\sigma^{*} \mathrm{~N} 1-\mathrm{H} 3$ & 5,38 & 0,78 & 0,058 \\
\hline \multicolumn{5}{|c|}{ Ângulo diedro de $110^{\circ}$} \\
\hline NBO doador (i) & NBO receptor $(\mathrm{j})$ & $\Delta \mathrm{E}^{2} \mathrm{kcal} \mathrm{mol}^{-1}$ & $\varepsilon_{\mathrm{i}}-\varepsilon_{\mathrm{j}}$ & $F(i, j)$ u.a \\
\hline$\sigma \mathrm{N} 1-\mathrm{H} 2$ & $\sigma^{*} \mathrm{~N} 1-\mathrm{N} 4$ & 0,53 & 0,88 & 0,019 \\
\hline$\sigma \mathrm{N} 1-\mathrm{H} 2$ & $\sigma^{*} \mathrm{~N} 4-\mathrm{H} 5$ & 0,99 & 1,09 & 0,029 \\
\hline$\sigma \mathrm{N} 1-\mathrm{H} 3$ & $\sigma^{*} \mathrm{~N} 1-\mathrm{N} 4$ & 0,53 & 0,88 & 0,019 \\
\hline$\sigma \mathrm{N} 1-\mathrm{H} 3$ & $\sigma^{*} \mathrm{~N} 4-\mathrm{H} 6$ & 0,99 & 1,09 & 0,029 \\
\hline$\sigma \mathrm{N} 4-\mathrm{H} 5$ & $\sigma^{*} \mathrm{~N} 1-\mathrm{H} 2$ & 0,99 & 1,09 & 0,029 \\
\hline$\sigma \mathrm{N} 4-\mathrm{H} 5$ & $\sigma^{*} \mathrm{~N} 1-\mathrm{N} 4$ & 0,53 & 0,88 & 0,019 \\
\hline$\sigma \mathrm{N} 4-\mathrm{H} 6$ & $\sigma^{*} \mathrm{~N} 1-\mathrm{H} 3$ & 0,99 & 1,09 & 0,029 \\
\hline$\sigma \mathrm{N} 4-\mathrm{H} 6$ & $\sigma^{*} \mathrm{~N} 1-\mathrm{N} 4$ & 0,53 & 0,88 & 0,019 \\
\hline $\mathrm{n} \pi \mathrm{N} 1$ & $\sigma^{*} \mathrm{~N} 4-\mathrm{H} 5$ & 1,39 & 0,79 & 0,030 \\
\hline $\mathrm{n} \pi \mathrm{N} 1$ & $\sigma^{*} \mathrm{~N} 4-\mathrm{H} 6$ & 1,39 & 0,79 & 0,030 \\
\hline $\mathrm{n} \pi \mathrm{N} 4$ & $\sigma^{*} \mathrm{~N} 1-\mathrm{H} 2$ & 1,39 & 0,79 & 0,030 \\
\hline $\mathrm{n} \pi \mathrm{N} 4$ & $\sigma^{*} \mathrm{~N} 1-\mathrm{H} 3$ & 1,39 & 0,79 & 0,030 \\
\hline \multicolumn{5}{|c|}{ Ângulo diedro de $290^{\circ}$} \\
\hline NBO doador (i) & NBO receptor $(\mathrm{j})$ & $\Delta \mathrm{E}^{2} \mathrm{kcal} \mathrm{mol}^{-1}$ & $\varepsilon_{\mathrm{i}}-\varepsilon_{\mathrm{j}}$ & $F(i, j)$ u.a \\
\hline$\sigma \mathrm{N} 1-\mathrm{H} 2$ & $\sigma^{*} \mathrm{~N} 4-\mathrm{H} 6$ & 2,16 & 1,08 & 0,043 \\
\hline$\sigma \mathrm{N} 1-\mathrm{H} 3$ & $\sigma^{*} \mathrm{~N} 4-\mathrm{H} 5$ & 2,16 & 1,08 & 0,043 \\
\hline$\sigma \mathrm{N} 4-\mathrm{H} 5$ & $\sigma^{*} \mathrm{~N} 1-\mathrm{H} 3$ & 2,16 & 1,08 & 0,043 \\
\hline$\sigma \mathrm{N} 4-\mathrm{H} 6$ & $\sigma^{*} \mathrm{~N} 1-\mathrm{H} 2$ & 2,16 & 1,08 & 0,043 \\
\hline
\end{tabular}

\section{Estrutura eletrônica da barreira rotacional da hidrazina (Análise QTAIM da hidrazina)}

A análise QTAIM foi empregada para avaliar os parâmetros topológicos das conformações da hidrazina. Estes parâmetros estudados são a densidade eletrônica $(\rho)$, a laplaciana da densidade eletrônica $\left(\nabla^{2} \rho\right)$ e a elipticidade $(\varepsilon)$, em que todos estes são em função de $r$, ou seja, da coordenada dos elétrons. Na Tabela 2 estão representados os valores $\rho(\mathrm{r}), \nabla^{2} \rho(\mathrm{r})$ e $\varepsilon(\mathrm{r})$ para os confôrmeros em $30^{\circ}, 110^{\circ}$ e $290^{\circ}$.
Quanto maior for o valor de $\rho(\mathrm{r})$, maior será a densidade eletrônica no ponto crítico de ligação (BCP). $\nabla^{2} \rho(\mathrm{r})$ permite que seja verificado o tipo da ligação, ou seja, se é uma ligação eletrostática ou covalente, no qual, para $\nabla^{2} \rho(\mathrm{r})>0$, a ligação é do tipo eletrostática, enquanto que $\nabla^{2} \rho(\mathrm{r})<0$ indica que a ligação é covalente. $\varepsilon(\mathrm{r})$ permite verificar o caráter $\pi$, em que $\varepsilon(\mathrm{r})=0$ indica que a ligação é essencialmente $\sigma$ e quanto maior for o valor da $\varepsilon(\mathrm{r})$ maior será o caráter $\pi$, o que evidencia mecanismos de movimentação da densidade eletrônica na estrutura, uma vez 
que estas ligações, por serem $\sigma$, deveriam ter valores de $\varepsilon(\mathrm{r})=0$.

Fazendo uma análise geral das propriedades topológicas, tem-se que todo o valor da laplaciana de densidade eletrônica é menor que zero, evidenciando que todas as ligações são covalentes. A densidade eletrônica nas ligações $\mathrm{N}-\mathrm{H}$ varia muito pouco entre os confôrmeros, observando um leve decréscimo de $30^{\circ}$ para $110^{\circ}$ e um pequeno aumento de $110^{\circ}$ para $290^{\circ}$. A diminuição da densidade eletrônica nos BCPs de $30^{\circ}$ para $110^{\circ}$ indica uma movimentação da densidade eletrônica.

Tabela 2 - Resultados obtidos para a hidrazina, calculados em nível B3LYP/6-31+G(d,p) utilizando QTAIM nos diedros de $30^{\circ}, 110^{\circ}$ e $290^{\circ}$. Valores em ua.

\begin{tabular}{|c|c|c|c|c|c|}
\hline $\mathrm{BCP}$ & Nome & Átomos & $\rho(\mathrm{r})$ & $\nabla^{2} \rho(\mathrm{r})$ & $\varepsilon(\mathrm{r})$ \\
\hline \multicolumn{6}{|c|}{ Ângulo diedro de $30^{\circ}$} \\
\hline 1 & $\mathrm{BCP} 1$ & $\mathrm{~N} 1$ - N4 & 0,306318 & $-0,548599$ & 0,000656 \\
\hline 2 & ВCP2 & $\mathrm{N} 1-\mathrm{H} 2$ & 0,346751 & $-1,822155$ & 0,061851 \\
\hline 3 & ВСР3 & $\mathrm{N} 1-\mathrm{H} 3$ & 0,343055 & $-1,766394$ & 0,061163 \\
\hline 4 & BCP4 & $\mathrm{N} 4-\mathrm{H} 5$ & 0,346751 & $-1,822155$ & 0,061851 \\
\hline 5 & BCP5 & N4 - H6 & 0,343055 & $-1,766394$ & 0,061163 \\
\hline \multicolumn{6}{|c|}{ Ângulo diedro de $110^{\circ}$} \\
\hline 1 & BCP1 & $\mathrm{N} 1-\mathrm{N} 4$ & 0,279263 & $-0,471523$ & 0,136994 \\
\hline 2 & ВCP2 & $\mathrm{N} 1-\mathrm{H} 2$ & 0,342904 & $-1,770245$ & 0,054407 \\
\hline 3 & ВСР3 & $\mathrm{N} 1-\mathrm{H} 3$ & 0,342902 & $-1,770235$ & 0,054407 \\
\hline 4 & BCP4 & N4 - H5 & 0,342904 & $-1,770245$ & 0,054407 \\
\hline 5 & BCP5 & N4 - H6 & 0,342902 & $-1,770235$ & 0,054407 \\
\hline \multicolumn{6}{|c|}{ Ângulo diedro de $290^{\circ}$} \\
\hline 1 & BCP1 & $\mathrm{N} 1-\mathrm{N} 4$ & 0,276814 & $-0,459401$ & 0,102191 \\
\hline 2 & BCP2 & $\mathrm{N} 1-\mathrm{H} 2$ & 0,343174 & $-1,783224$ & 0,044385 \\
\hline 3 & ВСР3 & $\mathrm{N} 1-\mathrm{H} 3$ & 0,343174 & $-1,783219$ & 0,044385 \\
\hline 4 & BCP4 & N4 - H5 & 0,343174 & $-1,783224$ & 0,044385 \\
\hline 5 & BCP5 & N4 - H6 & 0,343174 & $-1,783219$ & 0,044385 \\
\hline
\end{tabular}

O resultado mais significativo é a variação da elipticidade na ligação $\sigma \mathrm{N}-\mathrm{N}$ de $30^{\circ}$ para $110^{\circ}$, em que os valores encontrados foram de 0,000656 ua para o diedro em $30^{\circ}$ e 0,136994 ua para o diedro em $110^{\circ}$. A alteração na elipticidade evidência um aumento no caráter $\pi$ da ligação em $110^{\circ}$, revelando a movimentação da densidade eletrônica. Este resultado corrobora com o encontrado pelo NBO em que os efeitos de movimentação da densidade eletrônica se sobressaem sobre a repulsão de Pauli. Para as estruturas nos diedros em $30^{\circ}$ e $290^{\circ}$, existe disputa entre esses fatores eletrônicos, em que a repulsão de Pauli é favorecida no diedro de $30^{\circ}$. Para as ligações $\mathrm{N}-\mathrm{H}$ há pouca variação na elipticidade mostrando, assim, o maior caráter sigma das ligações.

\section{Estrutura eletrônica da barreira rotacional da hidrazina (Análise EDA da hidrazina)}

Os resultados para a metodologia EDA foram realizadas e os resultados estão representados na Tabela 3. A Tabela 3 mostra a diferença de energia total $(\Delta E)$, e os descritores cinético $\left(\Delta T_{s}\right)$, de troca e correlação $\left(\Delta E_{x c}\right)$, eletrostático $\left(\Delta \mathrm{E}_{e}\right)$, HOMO $(\Delta \mathrm{HOMO})$, LUMO $(\Delta \mathrm{LUMO})$ e Gap $(\Delta \mathrm{Gap}=$ HOMO-LUMO).

Pelos resultados é possível inferir que para a primeira barreira rotacional $\left(10,01 \mathrm{kcal} \mathrm{mol}^{-1}\right)$ os descritores de energia cinética e LUMO apresentam valores negativos. É importante destacar que o termo cinético mostra a movimentação dos elétrons na mudança de conformação, ou seja, movimentação da densidade eletrônica.

Tabela 3 - Diferença de energia total e diferença dos componentes calculados pela EDA para a hidrazina. Valores em kcal mol${ }^{-1}$.

\begin{tabular}{|c|c|c|c|c|c|c|c|}
\hline $\begin{array}{l}\text { Barreira } \\
\text { Rotacional }\end{array}$ & $\Delta \mathrm{E}$ & $\Delta \mathrm{T}_{\mathrm{s}}$ & $\Delta \mathrm{E}_{e}$ & $\Delta \mathrm{E}_{\mathrm{xc}}$ & $\Delta \mathrm{HOMO}$ & $\triangle \mathrm{LUMO}$ & $\Delta$ Gap \\
\hline$\Delta\left(30^{\circ}-110^{\circ}\right)$ & 10,01 & $-1,46$ & 2,89 & 8,58 & 27,34 & $-6,87$ & 34,20 \\
\hline$\Delta\left(30^{\circ}-290^{\circ}\right)$ & 2,95 & 9,56 & $-14,23$ & 7,62 & 25,23 & 2,42 & 22,82 \\
\hline
\end{tabular}


Para a segunda barreira rotacional $(2,95 \mathrm{kcal}$ $\mathrm{mol}^{-1}$ ) apenas o descritor de energia eletrostática apresenta valor negativo evidenciando assim que o termo eletrostático na conformação em $290^{\circ}$ é mais significativo que em $30^{\circ}$ resultado da repulsão entre os átomos. Os valores encontrados ratificam os reportados para a análise NBO e QTAIM.

\section{Trabalhos da literatura (NBO)}

As aplicações de cálculos NBO podem ser evidenciadas em diversos artigos científicos e alguns trabalhos são discutidos para elucidar a aplicação deste método como por exemplo "What is a Hydrogen Bond? Resonance Covalency in the supramolecular domain" (Weinhold and Klein, 2014), "Rabbit Ears Hybrids, VSEPR Sterics, and other Orbital Anachronisms" (Clauss et al, 2014), "The NBO Views of Chemical Bonding" (Landis e Weinhold, 2014).

Gangadharan e Krishnanb (2013), estudaram em nível B3LYP/6-31G(d,p) as reações do 8-hidroxiquinolina em falta ou excesso de fluorescência em solução com água, ácidos concentrados, dentre outros. Os resultados do trabalho mostraram que os tipos de hibridização e a natureza da ligação do composto são ligações sigmas que são estruturadas a partir de híbrido $s p^{1.64}$ no átomo de nitrogênio e que a ligação $\pi$ possui hibridização $s p^{3.12}$. Ainda observaram que a doação de elétrons mais forte ocorre através da interação do orbital do par isolado de elétrons com o orbital antiligante do aceitador de ligação $\pi^{*}$ (Gangadharan e Krishnan, 2013).

Thakur e Desiraju (2007), fizeram um estudo teórico das interações $\mathrm{C}-\mathrm{H} \cdots \mathrm{M}$, em que $\mathrm{M}$ pode ser os respectivos elementos: Ferro (Fe), Escândio $(\mathrm{Sc})$, Titânio (Ti), Vanádio (V), Cromo $(\mathrm{Cr})$, Manganês $(\mathrm{Mn})$, Cobalto $(\mathrm{Co})$, Cobre $(\mathrm{Cu})$, Zinco $(\mathrm{Zn})$ e Níquel (Ni). Neste estudo as interações foram avaliadas usando as geometrias no nível B3LYP/6$31 \mathrm{G}(\mathrm{d}, \mathrm{p})$ com abordagem de análise NBO (Thakur e Desiraju, 2007). Os resultados forneceram uma medida quantitativa da deslocalização doador-receptor nas ligações covalentes entre um fragmento $\mathrm{C}-\mathrm{H}$ e uma deficiência de elétrons dos metais que se diferem pelo método NBO. Os resultados também evidenciaram que nas ligações de hidrogênio há uma distinção de interação intramolecular devido às semelhanças geométricas (Thakur e Desiraju, 2007).

\section{Trabalhos da literatura (QTAIM)}

A QTAIM tem sido aplicada em estudos envolvendo moléculas (Grabowski, 2012), aglomerados moleculares (Parthasarathi et al., 2005), cristais moleculares pequenos (Arputharaj et al., 2012;
Hathwar et al., 2011; Pavan et al., 2014) e emparelhamento e empilhamento de bases de DNA (Parthasarathi et al., 2004; Parthasarathi et al., 2005) entre outras aplicações. Independente do sistema químico, os estudos realizados a partir da QTAIM permitem a elucidação e explicação eletrônico-estrutural de várias tendências, como a caracterização e natureza das ligações de hidrogênio que comumente são encontradas em sistemas biológicos. Um exemplo da aplicação da QTAIM em ligações de hidrogênio é o trabalho de Lapointe et al. (2009) que estudou peptídeos helicoidais modelo$\mathrm{R}$ contendo todos os aminoácidos naturais, exceto a lisina e a arginina. Neste trabalho foi possível verificar que, algumas disposições da hélice podem ser explicadas a partir das ligações de hidrogênio, no qual, apenas argumentos hidrofóbicos e entrópicos não eram suficientes para evidenciar essas características. O estudo também permitiu evidenciar três tipos de ligações de hidrogênio que contribuem para a estabilidade de todos os peptídeos modelo.

As análises topológicas da QTAIM também podem ser empregadas no estudo de barreiras rotacionais e as preferências conformacionais de um dado sistema químico podem ser determinadas e justificadas em termos de estrutura eletrônica. Um exemplo dessas análises é um recém artigo publicado por de Aguiar Filho et al. (2019) em que foi estudado os efeitos estereoeletrônicos responsáveis pela altura das barreiras rotacionais do etano e derivados substituídos por flúor, cloro, bromo e iodo. Para o estudo QTAIM, o método MP2 (Møller e Plesset) foi empregado utilizando os conjuntos de base $6-31+\mathrm{G}(\mathrm{d}, \mathrm{p})$ para o etano e os derivados substituídos por flúor, cloro e bromo. Para os derivados contendo iodo, utilizou-se o conjunto 6-311G(d,p).

Juntamente com a análise QTAIM, foram realizadas análises NBO e EDA e as metodologias corroboraram entre si de tal forma que, para os etanos substituídos por flúor, em que a barreira rotacional decresce com a adição do mesmo, $\mathrm{o}$ aumento do caráter $\pi$ nas ligações $\mathrm{C}-\mathrm{F}$ favorecem efeitos de movimentação da densidade eletrônica, que pode ser hiperconjugativa ou de outra natureza. Já para os etanos substituídos por cloro, bromo e iodo, os resultados sugerem que o aumento da barreira rotacional para esses compostos se dá preferencialmente pela repulsão de Pauli, ou seja, efeito estérico, que aumenta conforme os mesmos são adicionados (de Aguiar Filho et al., 2019).

No mesmo sentido, Zhurov e Pinkerton (2015) fizeram uma análise experimental e teórica usando a QTAIM das interações inter e intramoleculares do ácido 2-nitrobenzóico cristalino, que consiste de um dímero com ligações de hidrogênio centrossimétricas. Os autores evidenciaram que as proprie- 
dades de densidade de carga, bem como as interações inter e intramoleculares se devem a ligações $\pi$ na ligação C-N girada com as de outros nitro-aromáticos coplanares. O empacotamento cristalino dos dímeros ligados pelo hidrogênio são dominadas pela interação $\mathrm{O} \cdots \mathrm{O}$ paralelas e perpendicularmente tem-se o domínio da interação $\mathrm{CH} \cdots \mathrm{O}$ (Zhurov e Pinkerton, 2015).

\section{Trabalhos da literatura (EDA)}

Algumas aplicações podem ser evidenciadas na literatura usando EDA, como o trabalho de Zhou et al. (2017) que estudaram a origem e a natureza do efeito anomérico. Os autores, usando EDA e suas modificações, conseguiram identificar e quantificar a diferença de energia axial-equatorial em ciclohexanos em que o efeito anomérico aparece como um caso em especial.

Em outro trabalho Su e Li (2009) usaram EDA para estudar diversos sistemas tais como: a formação da ligação covalente no etano, metano e no $\mathrm{H}_{2}$, a barreira rotacional do $\mathrm{CH}_{3} \mathrm{CH}_{3}$, ligações de hidrogênio na água e amônia, interações iônicas, interações de van der Waals dentre outras. Os resultados usando a EDA permitiu explicar todos os sistemas estudados e quantificar os efeitos esteroeletrônicos responsáveis por cada propriedade.

\section{CONCLUSÕES}

Os cálculos teóricos de estrutura eletrônica são mecanismos essenciais utilizados na química teórica. Sua importância varia desde a análises de rotação, hiperconjugação, efeitos estéricos à cálculos de localização de orbitais, nível de energia entre orbitais ligantes e antiligantes e efeitos esteroeletrônicos. As teorias como QTAIM, NBO e EDA, são exemplos de ferramentas que ajudam em tais análises.

A elucidação dos efeitos estereoeletrônicos regentes nas conformações foram realizados após a perfilação da barreira rotacional da molécula hidrazina que teve valores calculados para a barreira rotacional interna de $10,01 \mathrm{kcal} \mathrm{mol}^{-1} \mathrm{e} 2,95 \mathrm{kcal} \mathrm{mol}^{-}$ ${ }^{1}$ para o método B3LYP/6-31+G (d,p).

A metodologia NBO é utilizada na compreensividade dos orbitais ligante e antiligantes dos sistemas químicos. Foi possível identificar todos os orbitais que interagem e os resultados corroboram com a geometria do sistema nos diedros de mínimo e máximo.

Os resultados da QTAIM para hidrazina evidenciaram que quando ocorre a alteração do confôrmero de $30^{\circ}$ até o de $110^{\circ}$ há um decréscimo da densidade eletrônica, evidenciando a interação en- tre orbitais e um acréscimo entre $110^{\circ}$ e $290^{\circ}$. Havendo uma relevância na variação da elipticidade, teve-se que na ligação N-N aumentou-se o caráter $\pi$ no confôrmero em $110^{\circ}$, a não relevância nesta variação, como no caso das ligações $\mathrm{N}-\mathrm{H}$, é evidenciado um caráter $\sigma$.

Para a EDA os resultados para a hidrazina mostraram que para a primeira barreira rotacional, revelou-se uma movimentação da densidade eletrônica devido à mudança de conformação, evidenciado pelo termo cinético, já para a segunda barreira rotacional, o valor encontrado para o termo eletrostático revela que este é o termo mais significativo para essa barreira, fruto da repulsão de Pauli.

Assim, o presente trabalho objetivou-se em uma breve explicação das abordagens das teorias citadas, trazendo aplicações das mesmas no sistema químico hidrazina e ainda em aplicações teóricas encontradas na literatura que elucidam os efeitos estereoeletrônicos.

\section{AGRADECIMENTO}

Os autores agradecem ao Centro Nacional de Processamento de Alto Desempenho (CENAPAD), em São Paulo, UNICAMP (Universidade Estadual de Campinas) e à FAPESP (Fundação de Amparo à Pesquisa do Estado de São Paulo - Centro de Engenharia e Ciências da Computação, Grant 2013/08293-7 e Grant 2017/11485-6) pelos recursos computacionais. Os autores também agradecem o financiamento da CAPES (Coordenação de Aperfeiçoamento de Pessoal de Nível Superior - Brasil), Código de Financiamento 001 CAPES.

\section{REFERÊNCIAS BIBLIOGRÁFICAS}

Anderson JSM. et al. Molecular QTAIM Topology Is Sensitive to Relativistic Corrections. Chemistry - A European Journal, v.25, n.10, p.2538-2544, 2019.

https://doi.org/10.1002/chem.201804464

Arputharaj DS. et al. Topological Electron Density Analysis and Electrostatic Properties of Aspirin: An Experimental and Theoretical Study. Crystal Growth \& Design, v.12, n.9, p.4357-4366, 2012.

https://doi.org/10.1021/cg300269n

Bader RFW. Atoms in molecules. Accounts of Chemical Research, v.18, n.1, p.9-15, 1985.

Bader RFW, Nguyen-Dang TT. Quantum Theory of Atoms in Molecules-Dalton Revisited. In: LÖWDIN, P.-O. Advances in Quantum Chemistry. Academic Press, v.14, p.63-124, 1981.

Clauss AD, Nelsen SF, Ayoub M, Moore JW, Landis CR, Weinhold F. "Rabbit Ears Hybrids, VSEPR Sterics, and Other Orbital Anachronisms", Chem. Educ. Res. Pract., v.15, p.417-434, 2014. 
Cukrowski, I. IQA-embedded fragment attributed molecular system energy change in exploring intramolecular interactions. Computational and Theoretical Chemistry, v.1066, p.62-75, 2015.

https://doi.org/10.1016/j.comptc.2015.04.018

De Aguiar Filho SQ. et al. Theoretical study of the internal rotational barriers of fluorine, chlorine, bromine, and iodine-substituted ethanes. Computational and Theoretical Chemistry, v.1166, p.112589, 2019.

https://doi.org/10.1016/j.comptc.2019.112589

Dennington R, Keith T, Millam J. (2009) Gauss View, Version 5. Semichem Inc., Shawnee Mission.

Dreizler RM, Gross EKU. Density Functional Theory: An Approach to the Quantum Many-Body Problem. SpringerVerlag, New York, 1990.

Foster JP, Weinhold F. Natural hybrid orbitals. Journal of the American Chemical Society, v.102, n.24, p.7211-7218, 1980.

Frisch MJ, Trucks GW, Schlegel HB, Scuseria GE, Robb MA, Cheeseman JR, Scalmani G, Barone V, Mennucci B, Petersson GA, Nakatsuji H, Caricato M, LI X, Hratchian, HP, Izmaylov AF, Bloino J, Zheng G, Sonnenberg JL, Hada M, Ehara M, Toyota K, Fukuda R, Hasegawa J, Ishida M, Nakajima T, Honda Y, Kitao O, NakaI H, Vreven T, Montgomery JA, Peralta JR, Ogliaro F, Bearpark M, Heyd JJ, Brothers E, Kudin KN, Staroverov VN, Kobayashi R, Normand J, Raghavachari K, Rendell A, Burant JC, Iyengar SS, Tomasi J, Cossi M, Rega N, Millam JM, Klene M, Knox JE, Cross JB, Bakken V, Adamo C, Jaramillo J, Gompert R, Stratmann RE, Yazyev O, Austin AJ, Cammi R, Pomelli C, Ochterski JW, Martin RL, Morokuma K, Zakrzewski VG, Voth GA, Salvador P, Dannenberg JJ, Dapprich S, Daniels AD, Farkas Ö, Foresman JB, Ortiz JV, Cioslowski J, Fox DJ. Gaussian 09, REVISION D.01, GAUSSIAN, INC.: WALLINGFORD CT, 2009.

Gangadharan P, Krishnan S. Physica polonica a natural bond orbital (NBO) Population Analysis of 1-Azanapthalene-8ol Rubarani, v.121, n.1, 2013. https://doi.org/10.12693/APhysPolA.125.18

Glendening ED, Landis CR, Weinhold F. Natural bond orbital methods. WIREs Computational Molecular Science, v.2, n.1, p.1-42, 2012.

Grabowski SJ. What Is the Covalency of Hydrogen Bonding? Chemical Reviews, v.111, n.4, p.2597-2625, 2011. https://doi.org/10.1021/cr800346f

Hathwar VR. et al. Charge Density Analysis of a Pentaborate Ion in an Ammonium Borate: Toward the Understanding of Topological Features in Borate Minerals. The Journal of Physical Chemistry A, v.115, n.45, p.12818-12825, 2011. https://doi.org/10.1021/jp203983v

Hehre WJ. Ab initio molecular orbital theory. Accounts of Chemical Research, v.9, n.11, p.399-406, 1976. https://doi.org/10.1021/ar50107a003

Juaristi E, Cuevas G. Recent studies of the anomeric effect. Tetrahedron, v.48, n.24, p.5019-5087, 1992.
Khaliullin RZ. et al. Unravelling the Origin of Intermolecular Interactions Using Absolutely Localized Molecular Orbitals. The Journal of Physical Chemistry A, v.111, n.36, p.8753-8765, 2007.

Kumar PSV, Raghavendra V, Subramanian V. Bader's Theory of Atoms in Molecules (AIM) and its Applications to Chemical Bonding. Journal of Chemical Sciences, v.128, n.10, p.1527-1536, 2016. https://doi.org/10.1007/s12039-016-1172-3

Landis CR, Weinhold F. "The NBO View of Chemical Bonding", in, G. Frenking and S. Shaik (eds.), The Chemical Bond: Fundamental Aspects of Chemical Bonding, p. 91120, 2014.

Lapointe SM. et al. QTAIM study of an alpha-helix hydrogen bond network. The Journal of Physical Chemistry. B, v.113, n.31, p.10957-10964, 2009. https://doi.org/10.1021/jp903635h

Liu S. Origin and Nature of Bond Rotation Barriers: A Unified View. The Journal of Physical Chemistry A, v.117, n.5, p.962-965, 2013. https://doi.org/10.1021/jp312521z

Liu S. Steric effect: A quantitative description from density functional theory. The Journal of Chemical Physics, v.126, n.24, p.244103, 2007. https://doi.org/10.1063/1.2747247

Liu S, Govind N, Pedersen LG. Exploring the origin of the internal rotational barrier for molecules with one rotatable dihedral angle. The Journal of Chemical Physics, v.129, n.9, p.094104, 2008. https://doi.org/10.1063/1.2976767

Liu S, Rong C, Lu T. Electronic forces as descriptors of nucleophilic and electrophilic regioselectivity and stereoselectivity. Physical Chemistry Chemical Physics, v.19, n.2, p.1496-1503, 2017. https://doi.org/10.1039/C6CP06376D

Liu S, Schauer CK. Origin of molecular conformational stability: Perspectives from molecular orbital interactions and density functional reactivity theory. The Journal of Chemical Physics, v.142, n.5, p.054107, 2015. https://doi.org/10.1063/1.4907365

Löwdin PO. Quantum Theory of Many-Particle Systems. I. Physical Interpretations by Means of Density Matrices, Natural Spin-Orbitals, and Convergence Problems in the Method of Configurational Interaction. Physical Review, v.97, n.6, p.1474-1489, 1955. https://doi.org/10.1103/PhysRev.97.1474

Mitoraj MP, Michalak A, Ziegler TA. Combined Charge and Energy Decomposition Scheme for Bond Analysis. Journal of Chemical Theory and Computation, v.5, n.4, p.962-975, 2009.

https://doi.org/10.1021/ct800503d

Møller CHR, Plesset MS. Note on an Approximation Treatment for Many-Electron Systems. Physical Review, v.46, n.7, p.618-622, 1934.

Mitoraj M, Michalak A. Natural orbitals for chemical valence as descriptors of chemical bonding in transition metal complexes. Journal of Molecular Modeling, v.13, n.2, p.347355, 2007.

https://doi.org/10.1007/s00894-006-0149-4 
Morgon NH, Custodio R. Teoria do Funcional de Densidade. Química Nova (impress), v.18, n.1, p.44-55, 1995

Morokuma, K. Molecular Orbital Studies of Hydrogen Bonds. III. C $=\mathrm{O} \cdots \mathrm{H}-\mathrm{O}$ Hydrogen Bond in $\mathrm{H} 2 \mathrm{CO} \cdots \mathrm{H} 2 \mathrm{O}$ and $\mathrm{H} 2 \mathrm{CO} \cdot 2 \mathrm{H} 2 \mathrm{O}$. The Journal of Chemical Physics, v.55, n.3, p.1236-1244, 1971.

Mulliken RS, Rieke CA, Brown WG. Hyperconjugation*. Journal of the American Chemical Society, v.63, n.1, p.4156,1941

Oliveira BG, Araújo RCMU, Ramos MN. A topologia molecular QTAIM e a descrição mecânico-quântica de ligações de hidrogênio e ligações de di-hidrogênio. Química Nova, v.33, n.5, p.1155-1162, 2010 . https://doi.org/10.1590/S0100-40422010000500029

Parr RG, Yang W. Density-Functional Theory of Atoms and Molecules, Oxford University Press, New York, 1989.

Parthasarathi R. et al. Bader's and Reactivity Descriptors' Analysis of DNA Base Pairs. The Journal of Physical Chemistry A, v.108, n.17, p.3817-3828, 2004. https://doi.org/10.1021/jp031285f

Parthasarathi R, Subramanian V. Stacking Interactions in Benzene and Cytosine Dimers: From Molecular Electron Density Perspective. Structural Chemistry, v.16, n.3, p.243-255, 2005.

Parthasarathi R, Subramanian V, Sathyamurthy N. Hydrogen Bonding in Phenol, Water, and Phenol-Water Clusters. The Journal of Physical Chemistry A, v.109, n.5, p.843$850,2005$. https://doi.org/10.1021/jp046499r

Pavan MS. et al. Characterization of Interactions Involving Bromine in 2,2-Dibromo-2,3-dihydroinden-1-one via Experimental Charge Density Analysis. Crystal Growth \& Design, v.14, n.11, p.5477-5485, 2014. https://doi.org/10.1021/cg500659c

Pereira DH. et al. A study of the rotational barriers for some organic compounds using the G3 and G3CEP theories. Journal of Molecular Modeling, v.20, n.4, p.2199, 2014.

Popelier PLA. Quantum Chemical Topology: on Bonds and Potentials. In: WALES, D. J. (Ed.). Intermolecular Forces and Clusters I. Berlin, Heidelberg: Springer Berlin Heidelberg, v.115, p.1-56, 2005.

Popelier PLA, Bader RFW. The existence of an intramolecular C-H-O hydrogen bond in creatine and carbamoyl sarcosine. Chemical Physics Letters, v.189, n.6, p.542-548, 1992.

Raissi, H. et al. Intramolecular Hydrogen Bonding in Structural Conformers of 2-Amino Methylene Malonaldehyde: AIM and NBO Studies. International Journal of Quantum Chemistry, v.110, p.821-830, 2009.

https://doi.org/10.1002/qua.21795

Reis D, Ribeiro I, Pereira DH. DFT study of the application of polymers cellulose and cellulose acetate for adsorption of metal ions $(\mathrm{Cd} 2+, \mathrm{Cu} 2+$ and $\mathrm{Cr} 3+)$ potentially toxic. Polymer Bulletin, 2019.

Su P, Li H. Energy decomposition analysis of covalent bonds and intermolecular interactions. The Journal of Chemical Physics, v.131, n.1, p.014102, 2009. https://doi.org/10.1063/1.3159673
Thakur TS, Desiraju GR. Theoretical investigation of C$\mathrm{H} \cdots \mathrm{M}$ interactions in organometallic complexes: A natural bond orbital (NBO) study. Journal of Molecular Structure: THEOCHEM, v.810, n.1, p.143-154, 2007. https://doi.org/10.1016/j.theochem.2007.02.012

Todd A, Keith Aimall (Version 17.11.14), TK Gristmill Software, Overland Park KS, USA, 2017 (aim.tkgristmill.com)

Tsuboi M, Overend J. Amino wagging and inversion in hydrazines: RR branch of the antisymmetric wagging band of NH2NH2 J Mol Spectrosc, v.52, p.256-268, 1974.

Vener MV. et al. QTAIM Study of Strong H-Bonds with the $\mathrm{O}-\mathrm{H} \cdots$ A Fragment $(\mathrm{A}=\mathrm{O}, \mathrm{N})$ in Three-Dimensional Periodical Crystals. The Journal of Physical Chemistry A, v.111, n.6, p.1155-1162, 2007. https://doi.org/10.1021/jp067057d

Vorobyov I, Yappert MC, Dupré DB. Energetic and Topological Analyses of Cooperative $\sigma \mathrm{H}$ - and $\pi \mathrm{H}$-Bonding Interactions. The Journal of Physical Chemistry A, v.106, n.44, p.10691-10699, 2002. https://doi.org/10.1021/jp0264580

Weinhold FR, Klein R. "What is a Hydrogen Bond? Resonance Covalency in the Supramolecular Domain", Chem. Educ. Res. Pract. v.15, p.276-285, 2014. https://doi.org/10.1039/C4RP00030G

Weinhold F. Natural bond orbital analysis: A critical overview of relationships to alternative bonding perspectives. Journal of Computational Chemistry, v.33, n.30, p.23632379, 2012. https://doi.org/10.1002/jcc.23060

Zhao L. et al. Energy decomposition analysis. WIREs Computational Molecular Science, v.8, n.3, p.e1345, 2018.

Zhou X. et al. Anomeric effect revisited: Perspective from information-theoretic approach in density functional reactivity theory. Chemical Physics Letters, v.684, p.97-102, 2017.

Zhurov VV, Pinkerton AA. Inter- and Intramolecular Interactions in Crystalline 2-Nitrobenzoic Acid-An Experimental and Theoretical QTAIM Analysis. The Journal of Physical Chemistry A, v.119, n.52, p.13092-13100, 2015. https://doi.org/10.1021/acs.jpca.5b10027

Ziegler T, Rauk A. On the calculation of bonding energies by the Hartree Fock Slater method. Theoretica chimica acta, v.46, n.1, p.1-10, 1977. 